\title{
Disorderly Conduct of Benzamide IV: Crystallographic and Computational Analysis of High Entropy Polymorphs of Small Molecules
}

Noalle Fellah, ${ }^{\text {a }}$ Alexander G. Shtukenberg, ${ }^{*}$ a Eric J. Chan, ${ }^{\mathrm{b}}$ Leslie Vogt-Maranto, ${ }^{\mathrm{b}}$ Wenqian Xu, ${ }^{\mathrm{c}}$ Chao Li, ${ }^{a}$ Mark E. Tuckerman, ${ }^{*}$, b,d,e Bart Kahr, ${ }^{*}$, a Michael D. Ward ${ }^{*}$ a

aDepartment of Chemistry and Molecular Design Institute, New York University, New York City, NY, 10003, USA; 'bepartment of Chemistry, New York University, New York City, NY, 10003, USA; ${ }^{c} \mathrm{X}$-ray Science Division, Advanced Photon Source, Argonne National Laboratory, Argonne, Illinois, 60349, USA; ${ }^{\mathrm{d} C o u r a n t}$ Institute of Mathematical Science, New York University, New York City, NY, 10003, USA; ${ }^{\mathrm{e} N e w}$ York University-East China Normal University Center for Computational Chemistry at NYU Shanghai, 3663 Zhongshan Road North, Shanghai, 200062, China

\section{Supporting Information}

\section{TABLE OF CONTENTS}

Table S1. CPG manufacturer specifications.

Figure S1. Powder diffraction pattern of benzamide Form IV collected with Bruker D8 Discover GADDS microdiffractometer.

Table S2. Space groups selected for 2D simulated annealing.

Figure S2. Annotated synchrotron powder diffraction pattern of Form IV.

Figure S3. Comparison of synchrotron powder diffraction patterns of Form IV.

Figure S4. Energy vs. molecular volume plot for the predicted crystal structures.

Table S3. DFT optimized structures with low symmetry.

Table S4. Structural motifs found in candidate structures.

Table S5. Dihedral angles for internal contacts for best candidate Form IV structures.

Figure S5. Comparison of calculated powder diffraction patterns of best candidate Form IV structures.

Figure S6. Constructed $P c a 2_{1}$ structure with $\mathrm{I}_{\mathrm{V}}$-contact tiling motif.

\section{Crystallographic Information Files including with manuscript}

benzamide_CSP_GAFF.cif: Five-hundred lowest energy structure from CSP.

benzamide_2D_cell.cif: Thirty-five 2D cells from the initial CSP search consistent with the experimental lattice parameters for $h k 0$ indices.

benzamideIVcandidates_DFTOpt 3D.cif Seventeen 3D structures CSP and simulating annealing corresponding to entries in Table 1 and Table S2. 
Table S1. CPG pore manufacturer specifications.

\begin{tabular}{ccc}
\hline Pore size (nm) & $\mathbf{\pm \%}$ & Pore volume $\left(\mathbf{c m}^{\mathbf{3}} / \mathbf{g}\right)$ \\
\hline 4 & 12.1 & 0.38 \\
\hline 8 & 13.4 & 0.51 \\
\hline 35 & 10 & 1.49 \\
\hline 50 & 10 & 1.07 \\
\hline 100 & 10 & 1.11 \\
\hline 200 & 10 & 0.98
\end{tabular}

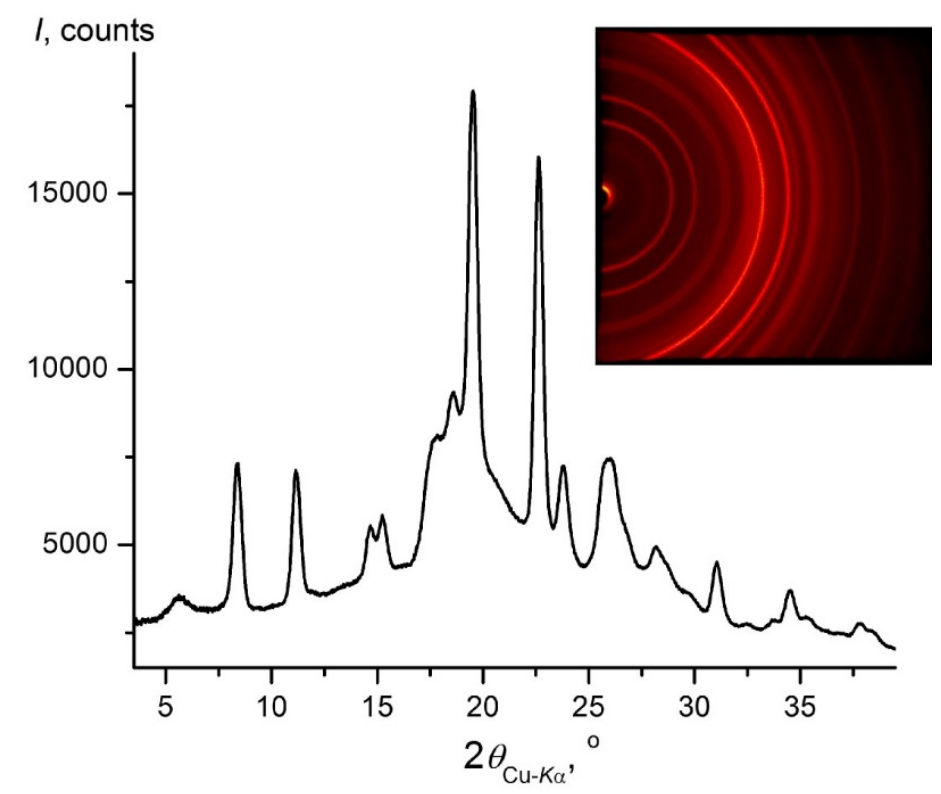

Figure S1. Powder diffraction pattern of benzamide IV (the starting material contains ca. $20 \mathrm{wt} \%$ of gum mastic) collected with Bruker D8 Discover GADDS microdiffractometer equipped with 2D detector (the original $2 \mathrm{D}$ pattern is shown as well). The peak at ca. $5.5^{\circ}$ corresponds to scattering from Kapton capillary. 
Table S2. Space groups selected for 2D simulated annealing. Structures with best $R_{w p}(\leq 12 \%)$ were selected as candidate models for structure solution.

\begin{tabular}{ccc}
\hline Space Group & $\boldsymbol{Z}$ & $\boldsymbol{R}_{\boldsymbol{w p}} \mathbf{( \% )}$ \\
\hline$P{ }_{1}{ }_{1} 2_{1}$ & 2 & 8.98 \\
$P 2_{1}{ }_{1} 2$ & 2 & 15.27 \\
$P n a 2_{1}$ & 2 & 10.48 \\
$P b a 2$ & 2 & 10.63 \\
$P b n 2_{1}$ & 2 & 13.55 \\
$P n n 2$ & 2 & 8.99 \\
$P b a m$ & 1 & 28.75 \\
$P n n m$ & 1 & 28.70 \\
$P b n m$ & 1 & 28.73 \\
$P n a m$ & 1 & 28.71 \\
$P 2_{1} / b 11$ & 2 & 13.68 \\
$P 12{ }_{1} / n 1$ & 2 & 10.59 \\
$P 12{ }_{1} / c 1^{\mathrm{a}}$ & 2 & 12.23 \\
\hline
\end{tabular}

a Space group setting selected after CSP

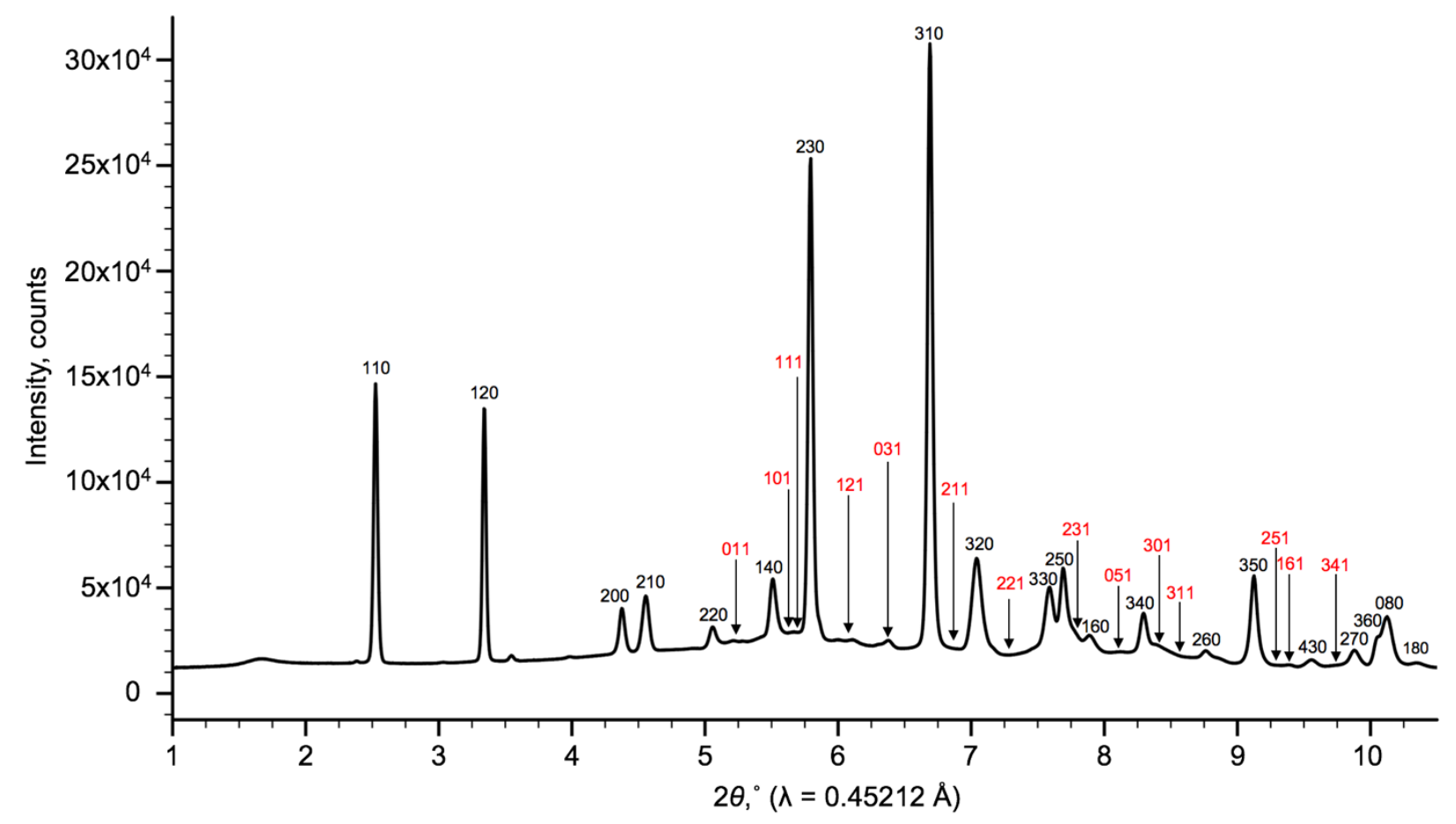

Figure S2. 17-BM beamline synchrotron diffraction data annotated with $h \mathrm{k} /$ indices. The unit cell is $a=11.83 \AA, b=$ $20.50 \AA$, and $c=5.03 \AA$ in the orthorhombic space group $P 2{ }_{1} 2_{1} 2_{1}$. Red indices indicate positions of $h k /$ indices where $I \neq 0$. 


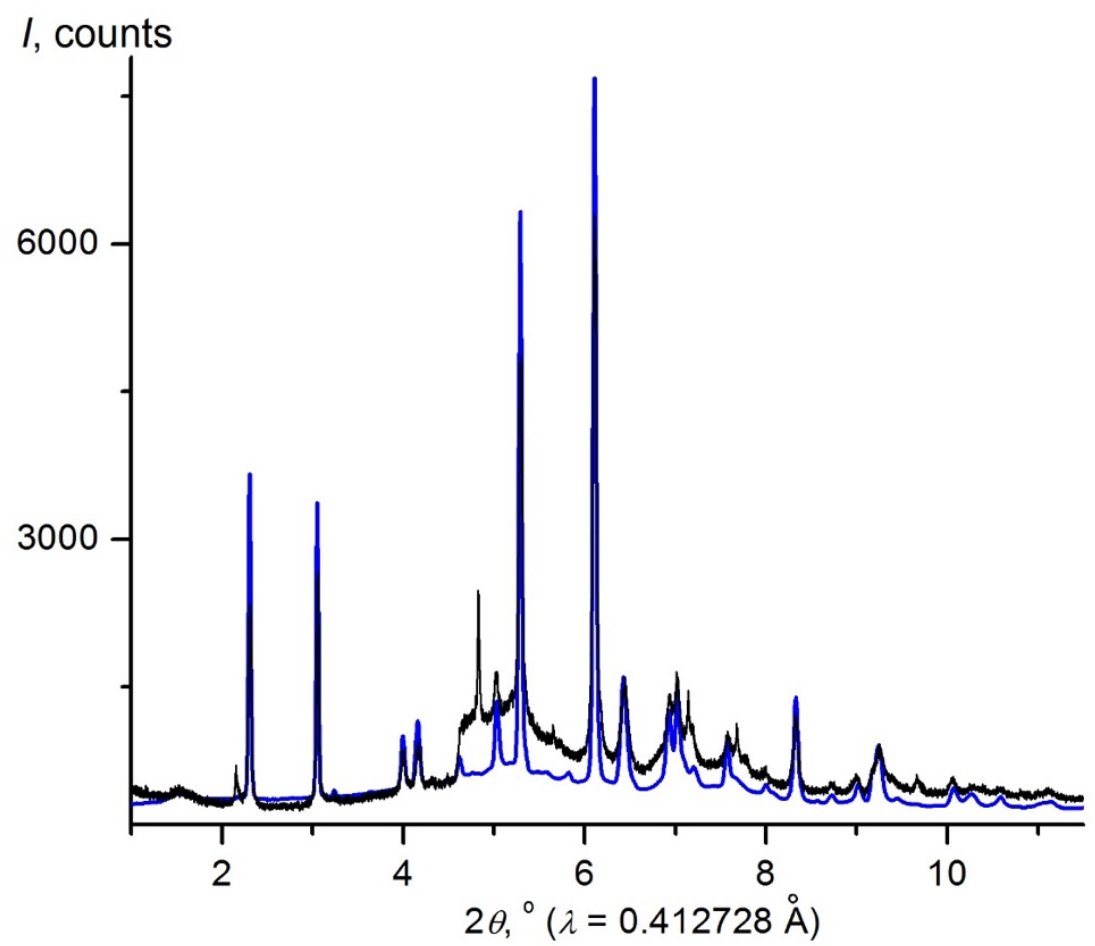

Figure S3. Comparison of synchrotron powder diffraction patterns of Form IV. Although 11-BM beamline data (black line) have higher angular resolution compared to 17-BM beamline data (blue line), the peak broadening is very similar in both datasets. All peaks of Form II are the same in both PXRD patterns. The observed differences are related to minor contamination by Forms II and III in the data from 17-BM (blue) and 11-BM (black) beamlines, respectively. 


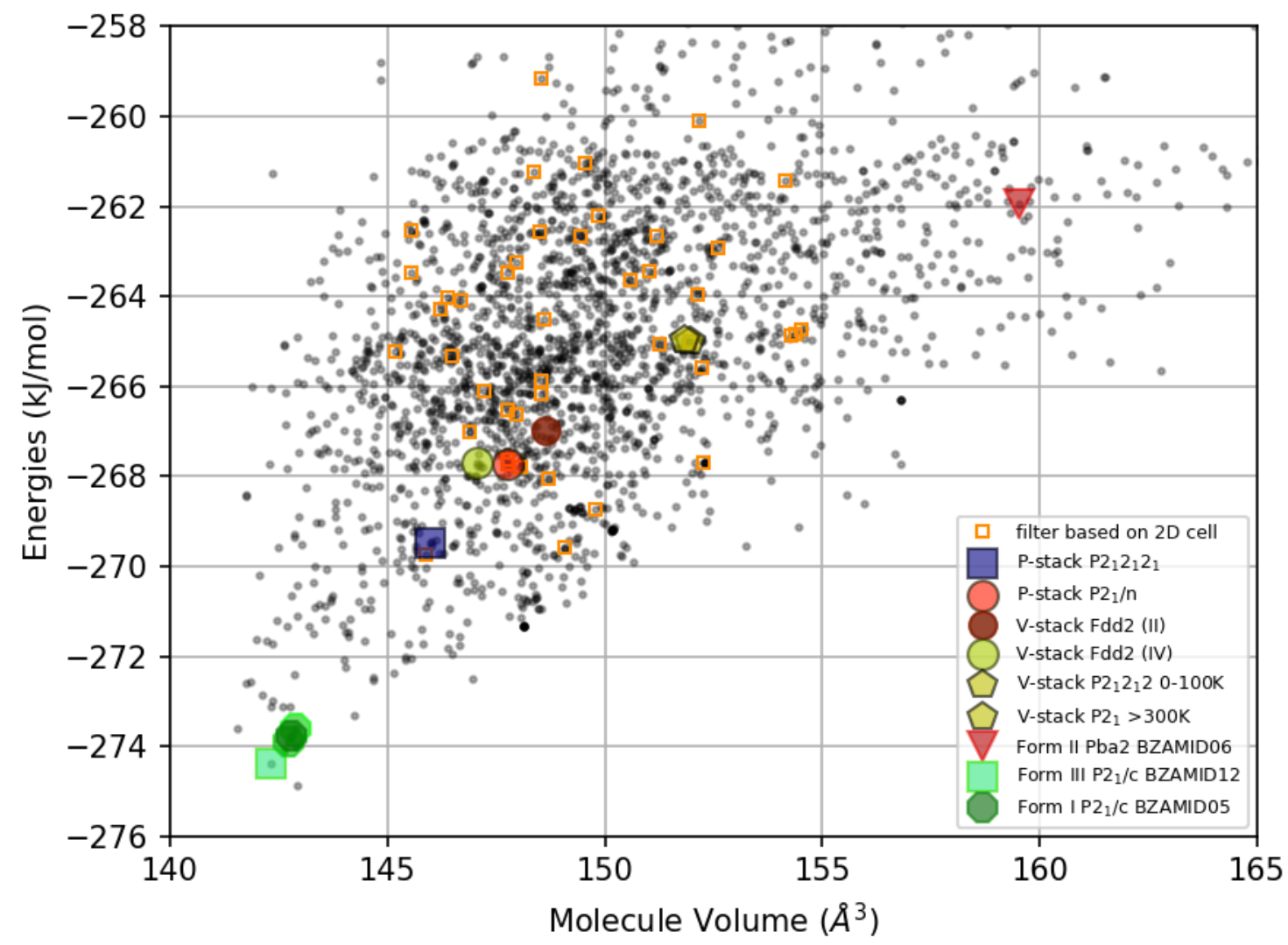

Figure S4. Energy vs. molecular volume plot for the predicted crystal structures (black dots). Those structures which had cell parameters within a 5\% tolerance of the 2D unit cell (indexed from PXRD) are indicated with orange boxes. Select previously reported forms and Form IV candidate structures are indicated by different markers, with multiple matching structures shown by varied shades of the same color and shape. Structures in the space group Fdd2 have been positioned into the plot manually by using the geometry optimization results derived from full-packed unit cell representations placed into the space group $P 1$. This was because the capability to perform a CSP search within this space group had not currently been implemented into UPACK software framework.

Table S3. DFT-optimized unit cell parameters and relative lattice energies for candidate Form IV structures with low symmetry

\begin{tabular}{ccccccccccc}
\hline $\begin{array}{c}\text { Generation } \\
\text { method }\end{array}$ & \multicolumn{2}{c}{ Space group } & \multirow{2}{*}{$\boldsymbol{Z}$} & $\boldsymbol{a}(\AA)$ & $\boldsymbol{b}(\AA)$ & $\boldsymbol{c}(\boldsymbol{\AA})$ & $\boldsymbol{\alpha}\left({ }^{\circ}\right)$ & $\boldsymbol{\beta}\left({ }^{\circ}\right)$ & $\boldsymbol{\gamma}\left({ }^{\circ}\right)$ & $\begin{array}{c}\boldsymbol{E} \\
(\mathbf{k J} / \mathbf{m o l})\end{array}$ \\
\hline SA & $P n n 2$ & $P 1$ & 8 & 5.195 & 11.758 & 21.222 & 89.48 & 88.05 & 77.34 & 7.26 \\
SA & $P b a 2$ & $P 1$ & 8 & 5.388 & 13.806 & 16.401 & 90.30 & 90.12 & 90.10 & 7.86 \\
SA & $P b a 2$ & $P 1$ & 8 & 5.192 & 11.716 & 20.886 & 90.00 & 94.06 & 90.76 & 7.90
\end{tabular}


Table S4. Hydrogen bonding and phenyl arrangement motifs found in candidate structures with tetrad packing

\begin{tabular}{ccccc}
\hline E (kJ/mol) & Space group & $\boldsymbol{Z}$ & Dimer/Catemer & $\begin{array}{c}\text { Internal phenyl } \\
\text { stacks, } \\
\text { IV or IP }\end{array}$ \\
\hline 0.00 & $P 2_{1} / c$ & 1 & $\mathrm{D}$ & $\mathrm{N} / \mathrm{A}$ \\
0.23 & $P 2_{1} / n$ & 1 & $\mathrm{D}$ & $\mathrm{N} / \mathrm{A}$ \\
2.52 & $P 2_{1} / n$ & 2 & $\mathrm{D}$ & $\mathrm{IV}$ \\
2.78 & $F d d 2$ & 2 & $\mathrm{C}$ & $\mathrm{IV}$ \\
4.48 & $F d d 2$ & 2 & $\mathrm{C}$ & $\mathrm{IV}$ \\
4.68 & $P 2_{1} 2_{1} 2_{1}$ & 2 & $\mathrm{C}$ & $\mathrm{IP}$ \\
6.20 & $P 2_{1} / c$ & 2 & $\mathrm{D}$ & $\mathrm{IV}$ \\
7.26 & $P 1$ & 8 & $\mathrm{C}$ & $\mathrm{IV}$ \\
7.44 & $P 2_{1} / n$ & 2 & $\mathrm{C}(\mathrm{anti})$ & $\mathrm{I}$ \\
7.86 & $P 1$ & 8 & $\mathrm{D}$ & $\mathrm{IP}$ \\
7.90 & $P 1$ & 8 & $\mathrm{D}$ & $\mathrm{IP}$ \\
8.21 & $P 2_{1} 2_{1} 2$ & 2 & $\mathrm{C}$ & $\mathrm{IV}$ \\
8.30 & $P c a 2_{1}$ & 4 & $\mathrm{C}$ & $\mathrm{IV}$ \\
8.98 & $P n a 2_{1}$ & 2 & $\mathrm{C}$ & $\mathrm{IP}$ \\
9.37 & $P 2_{1} 2_{1} 2_{1}$ & 2 & $\mathrm{D}$ & $\mathrm{IP}$ \\
10.24 & $P n a 2_{1}$ & 2 & $\mathrm{C}$ & $\mathrm{IP}$ \\
10.84 & $P 2_{1} / c$ & 2 & $\mathrm{D}$ & $\mathrm{IP}$ \\
\hline
\end{tabular}

Table S5. Structural parameters for candidate structures. Internal dihedral angles describe the geometry between closest phenyl rings within tiles.

\begin{tabular}{cccc}
\hline $\begin{array}{c}\text { Space groups } \\
\text { (Figure 7) }\end{array}$ & $\begin{array}{c}\text { Local tetrad } \\
\text { symmetry }\end{array}$ & $\begin{array}{c}\text { Internal dihedral } \\
\text { angle, } I_{\mathbf{v}}\left({ }^{\circ}\right)\end{array}$ & $\begin{array}{c}\text { Internal dihedral } \\
\text { angle, } \mathbf{I}_{\mathbf{P}}\left(^{\circ}\right)\end{array}$ \\
\hline$F d d 2$ & 2 & 67.1 & - \\
$P 2_{1} 22_{1}$ & 2 & 68.7 & - \\
$P 2_{1} / n$ & $\overline{1}$ & - & 9.5 \\
$P 2{ }_{1} 2_{1} 2_{1}$ & 2 & - & 11.4 \\
\hline
\end{tabular}




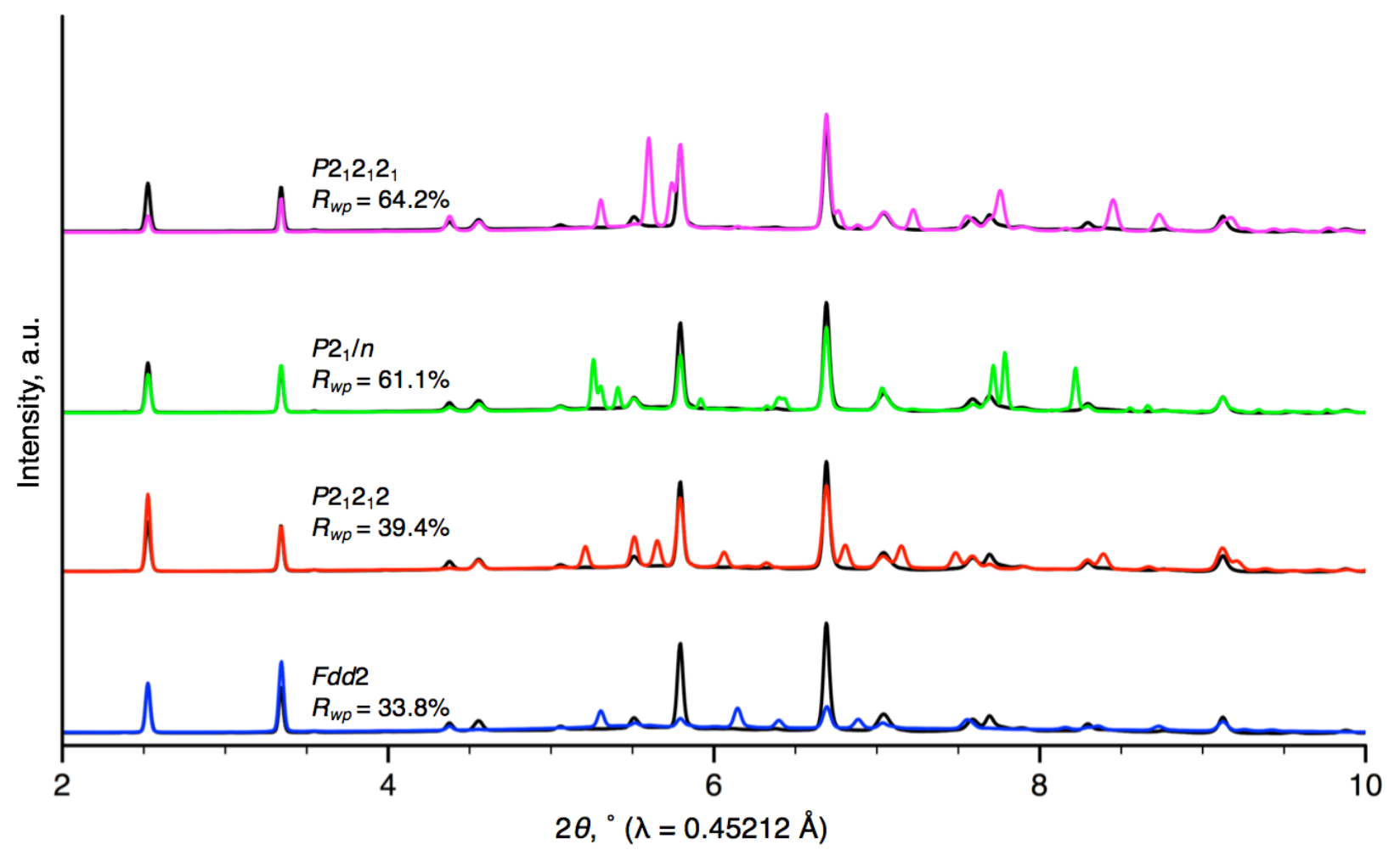

Figure S5. Overlay of calculated PXRD patterns of best candidate Form IV structures (color) and experimental PXRD data (black).

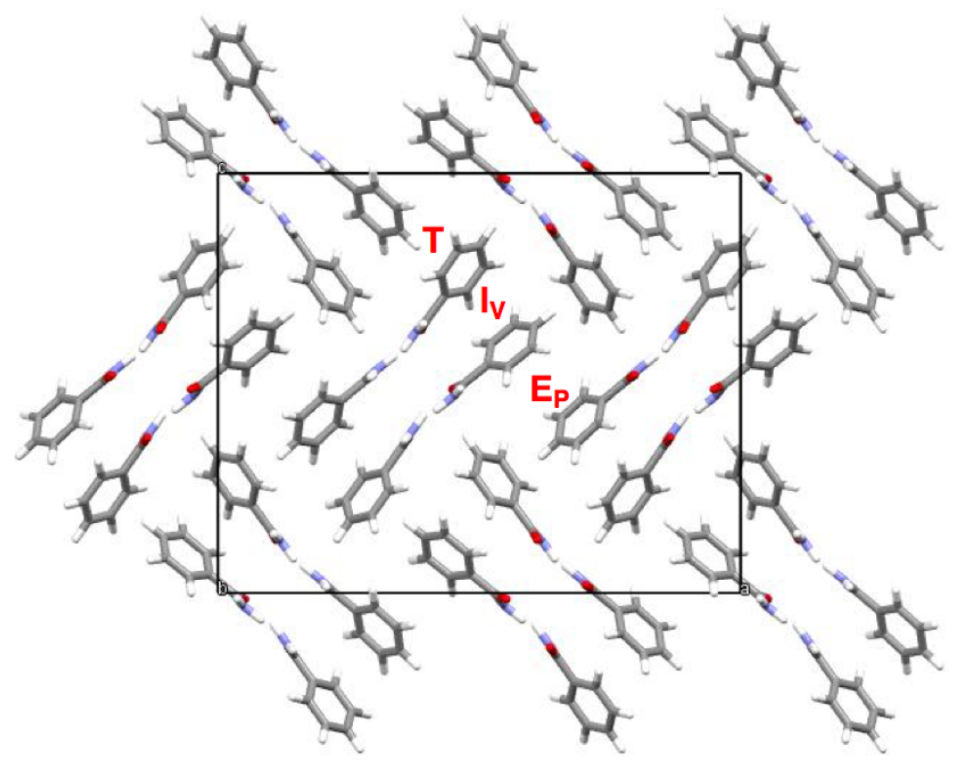

Figure S6. Projection of $P c a 2{ }_{1}$ structure $\left(Z^{\prime}=4 ; E=8.30 \mathrm{~kJ} / \mathrm{mol}\right)$. DFT optimized to unit cell with $a=$ $24.755 \AA, b=5.153 \AA, c=11.521 \AA$. Phenyl ring contacts are indicated and correspond to the following dihedral angles: $\mathrm{T}=68.4$ and $71.4^{\circ} ; \mathrm{E}_{\mathrm{P}}=6.5^{\circ} ; \mathrm{IV}=68.4$ and $71.4^{\circ}$. 